\title{
Pour une typologie de la marginalité dans les romans de Delly : la fonction de la femme
}

\author{
$\mathbf{M}^{\mathrm{a}}$ Teresa Lozano Sampedro \\ Universidad de Salamanca \\ tlozano@usal.es
}

Rebut: 15 de juny de 2018

Acceptat: 26 de juliol de 2018

RESUM

Sobre una tipologia de la marginalitat en les novel-les de Delly : la funció de la dona

L'obra de Delly (1875-1947), que s'inscriu en l'àmbit de la novel.la sentimental, presenta com una de les seves característiques principals la funció central de la dona. La situació inicial d'inferioritat de l'heroïna, sovint marcada per la pobresa, constitueix el punt de partida d'una intriga en què la jove, òrfena dotada de qualitats excepcionals - entre d'altres, la caritat envers els desafortunats- es trobarà envoltada de personatges secundaris diversos que exerciran el paper d'oponents o d'ajudants. Parentes pobres, geloses i venjatives, o de vegades parentes riques desdenyoses i ambicioses, són l'antítesi d'un altre tipus de dones: les criades velles, fidels i abnegades, el paper de les quals és indispensable per a aconseguir un final feliç de la història. En les tres novel-les que ens proposem analitzar -Esclave... ou reine (1910), La fin d'une Walkyrie (1916) i La Maison des Rossignols (1945) — l'etern maniqueïsme de la novel »la popular es manifesta a través d'una vasta tipologia.

Paraules Clau

Delly, novel·la sentimental, novel-la popular, dona, tipologia. 


\section{RÉSUMÉ}

\section{Pour une typologie de la marginalité dans les romans de Delly : la fonction de la femme}

L'œuvre de Delly (1875-1947), qui s'inscrit dans la ligne du roman sentimental, présente comme l'une de ses caractéristiques principales la fonction centrale de la femme. La situation initiale d'infériorité de l'hérö̈ne, souvent marquée par la pauvreté, constitue le point de départ d'une intrigue où la jeune fille, orpheline douée de qualités exceptionnelles - parmi d'autres, la charité envers les infortunés - se trouvera entourée de personnages secondaires divers qui exerceront le rôle d'opposants ou d'adjuvants. Des parentes pauvres, jalouses et vindicatives, ou parfois des parentes riches dédaigneuses et ambitieuses, sont l'antithèse d'un autre type de femmes : les servantes âgées, fidèles et dévouées, dont le rôle est indispensable pour la fin heureuse de l'histoire. Dans les trois romans que nous nous proposons d'analyser - Esclave ...ou reine ?(1910), La fin d'une Walkyrie (1916) et La Maison des Rossignols (1945) - l'éternel manichéisme du roman populaire se manifeste à travers une riche typologie.

\section{Mots Clés}

Delly, roman sentimental, roman populaire, femme, typologie.

\section{RESUMEN}

\section{Sobre una tipología de la marginalidad en las novelas de Delly : la función de la mujer}

La obra de Delly (1875-1947), que se inscribe en la línea de la novela sentimental, presenta como una de sus características principales la función central de la mujer. La situación inicial de inferioridad de la heroína, a menudo marcada por la pobreza, constituye el punto de partida de una intriga en que la joven, huérfana dotada de cualidades excepcionales - entre otras, la caridad para con los desafortunados - se encontrará rodeada de personajes secundarios diversos que ejercerán el papel de oponentes o de ayudantes. Parientes pobres, celosas y vengativas, o a veces parientes ricas desdeñosas y ambiciosas, son la antítesis de otro tipo de mujeres: las viejas sirvientes, fieles y abnegadas, cuyo papel es indispensable para el final feliz de la historia. En las tres novelas que nos proponemos analizar - Esclave... ou reine? (1910), La fin d'une Walkyrie (1916) y La Maison des Rossignols (1945) - el eterno maniqueísmo de la novela popular se manifiesta a través de una amplia tipología.

Palabras Clave

Delly, novela sentimental, novela popular, mujer, tipología. 


\begin{abstract}
For a Typology of Marginalization in Delly's Novels: Women's Function Delly's works (1875-1947), which join the line of sentimental novel, present women's central role as one of its main features. The original heroine's inferior status, often marked by poverty, is the starting point of a plot in which the young lady, an orphan, endowed with exceptional qualities - among others, charity towards the unfortunate -, will be surrounded by several secondary characters acting the part of opponents or assistants. Poor, jealous and vindictive female relatives, or even rich, disdainful and ambitious female siblings, are the antithesis of another type of women: the old, loyal and selfless servant, who plays a crucial role in leading the story to a happy ending. In the three novels that we intend to analyze - Esclave... ou reine? (1910), La fin d'une Valkyrie (1916) and La Maison des Rossignols (1945) - eternal popular novel Manichaeism appears through a broad typology.
\end{abstract}

\title{
KEY WORDS
}

Delly, sentimental novel, popular novel, women, typology.

\section{Introduction}

Delly est en principe le pseudonyme collectif de Jeanne Henriette Marie Petitjean de la Rosière (Avignon, 1875 - Versailles, 1947) et de son frère Frédéric Henri Joseph (Vannes, 1876 - Versailles, 1949). Sans établir la part de contribution de Frédéric dans l'immense production littéraire de l'œuvre - qui comprend plus de cent romans - groupée sous ce pseudonyme, c'est à l'écrivaine, à la femme, que nous ferons référence dans la présente étude. Son succès dans le genre du roman sentimental est incontestable : en 1914 elle avait déjà publié en France plus d'un tiers de son œuvre, et très longtemps après sa mort les publications de ses romans se sont succédées. La critique a souvent analysé les diverses raisons de ce succès, non seulement en France, mais dans d'autres pays et continents où les abondantes traductions des romans dellyens ont trouvé une réponse enthousiaste de la part des lecteurs. L'on pourrait donc s'interroger sur les sujets ou les motifs qui rendent si attrayante l'œuvre de l'auteure. Comme le signale Ellen Constans, en vue des dates des éditions : «L'œuvre de Delly a également marqué l'apogée et la fin du roman d'amour 
catholique militant $»^{1}$. En effet, d'un point de vue thématique, dans l'œuvre dellyenne la charité est souvent liée à la pratique de la religion catholique. Et le secours prêté par l'héroïne aux infortunés est l'un des éléments importants pour la métamorphose positive du héros masculin.

Dans les trois romans qui font l'objet de notre étude, l'héroïne présente cette caractéristique, dont l'importance varie cependant d'un roman à l'autre. Et elle se voit entourée de parents pauvres et de parents riches, qui exerceront tour à tour et sans distinction de classes le rôle d'opposants ou d'adjuvants. Le cadre de la narration présente une imprécision spatiale et temporelle, puisque les personnages de Delly ne nous laissent jamais oublier qu'ils sont des personnages de fiction, de manière que leur présence « loin de permettre une affectation temporelle précise de l'histoire, a un effet de déréalisation; nous sommes renvoyés aux pays des contes et légendes $»^{2}$. Il faut ajouter à cela le manichéisme inhérent au roman populaire, et aux contes de fées dont les récits de l'auteure suivent les schémas. Comme nous le verrons par la suite, en ce qui concerne les personnages secondaires il n'y a point de moyen terme entre les bons et les méchants. L'héroïne, jeune fille orpheline et appauvrie qui part d'une situation d'infériorité, en sera la victime ou la privilégiée, l'objet de la vengeance ou la protégée.

\section{La fonction des récits et la circularité : Esclave...ou Reine?}

L'un des attraits des romans de Delly consiste dans le recours à des schémas et des motifs appartenant à plusieurs genres, notamment au roman policier et au roman d'énigme. Cette inter-généricité tient en suspense l'attention du lecteur, toujours à l'attente de découvrir un mystère ou un secret dont des indices diffus sont suggérés dans les premières pages du récit : «Enlèvements, séquestrations, meurtres ou tentatives de meurtre [...] provoquent ou ont provoqué des mystères qu'il faudra éclaircir. La découverte des secrets et des énigmes et la révélation de l'amour s'accomplissent dans le même parcours textuel $»^{3}$.

\footnotetext{
${ }^{1}$ Ellen CONSTANS, Parlez-moi d'amour. Le roman sentimental : des romans grecs aux collections de l'an 2000, PULIM, Limoges, 1999, p. 236.

${ }^{2}$ Ellen CONSTANS, «Le Premier Delly ou comment l'idéologie subvertit le récit amoureux », Le Rocambole, (Bulletin des Amis du Roman populaire), Été- Automne 2011 : L'œuvre de Delly, Novelle série, ${ }^{\circ}$ 55-56, (pp. 45-81), p. 51.

${ }^{3}$ Ellen CONSTANS, Parlez-moi d'amour. Le roman sentimental : des romans grecs aux collections de l'an 2000, op. cit., p. 226.
} 
Esclave... ou Reine? (1910) constitue à nos yeux un exemple significatif de ce parcours parallèle. Comme il est fréquent dans les romans de l'écrivaine, le récit nous présente initialemen l'héroïne, la charmante jeune fille de 15 ans Lise de Subrans, qui vit avec sa belle-mère, Catherine, seconde épouse du vicomte de Subrans, qui était venu passer ses dernières années en Périgord avec sa famille «après avoir dissipé sa santé et sa fortune personnelle dans la grande vie parisienne $»^{4}$. Le motif de la méchante marâtre, présent dans d'autres romans de Delly, n'apparait pas pourtant dans Esclave... ou Reine? Catherine, qui ne cherche que le bonheur de sa belle-fille, est pour elle sa chère maman. C'est seulement au moment où le prince Serge Ormanoff - veuf âgé de trente ans, qui a perdu sa première femme, Olga, et son seul enfant dans des circonstances mystérieuses - exige à Catherine la main de Lise, encore adolescente, qu'un secret soigneusement gardé commence à se laisser entrevoir. Face à la résistance de Catherine à cette alliance, ce prince orgueilleux et dominateur n'hésite pas à prononcer une terrible menace : « Et ne pensezvous pas qu'elle sera plus à sa place près de moi, qui suis un honnête homme, que sous le toit de la femme qui a tué sa mère ? $»^{5}$. Catherine devra céder à ce chantage et Lise accepte un mariage qui lui fait horreur. Dans son nouvel état de femme mariée, où elle se trouvera perdue dans un étrange labyrinthe d'intrigues et de louches intérêts, elle ne se laisse pas éblouir par la splendide villa du prince à Cannes, car les hérö̈nes dellyennes restent insensibles à la richesse, d'autant plus que dans ces cadres luxueux elles ne seraient qu'un ornement. Les parures ne sont pour ces jeunes femmes, fortes et intègres, qu'un symbole d'esclavage, comme le bracelet offert, ou plutôt imposé par le prince à sa femme : «La princesse Ormanoff était une esclave, et le maître revendiquait jusqu'à la domination de sa conscience et de toute son âme $»^{6}$.

L'inégalité entre les sexes est une constante dans l'œuvre de Delly, et cela tient en grande partie à la différence d'âge entre l'homme et la femme : «Il est toujours plus âgé qu'elle; Il a l'expérience du monde et de la vie, Elle n'en a aucune ; Il est par nature le protecteur et le maître d'Elle » ${ }^{7}$. C'est bien le cas du roman qui nous occupe. Mais malgré cette domination de l'homme, dictée par les normes du roman traditionnel - « Les principes du catholicisme

\footnotetext{
${ }^{4}$ DELLY, Esclave... ou Reine ?, Librairie Plon, Paris, 1929, p. 10.

${ }^{5}$ Ibid., p. 40.

${ }^{6}$ Ibid., p. 109.

${ }^{7}$ Ellen CONSTANS, Parlez-moi d'amour. Le roman sentimental : des romans grecs aux collections de l'an 2000, op. cit., p. 231.
} 
le veulent ainsi $»^{8}-$ la singularité des héroïnes, mise en relief dès le début des romans dellyens, constituera leur force: «En règle générale l'héroïne est donnée comme quasi-parfaite dès les premières pages (ou le devient rapidement si elle est encore une préadolescente au début du texte) ${ }^{9}$. En effet, la beauté éblouissante de Lise, largement décrite dans le texte, est révélatrice " d'une délicatesse d'âme qu'aucun souffle délétère n'était venu effleurer ${ }^{10}$.

Si le passé des personnages principaux est toujours important dans les romans de Delly, il nous semble que dans Esclave ...ou Reine ? il joue une fonction spécialement remarquable. Le cadre initial du roman - qui est aussi le cadre final, comme nous le verrons plus tard - est le cimetière de la Bardonnaye où se trouve la tombe de Gabriel des Forcils, le très cher ami d'enfance de Lise, mort prématurément à l'âge de dix-huit ans. Gabriel a été le guide spirituel de la petite, éduquée par des parents indifférents en matière de religion. C'est lui, en définitive, qui a conduit sa chère amie vers la religion catholique et, par là, vers l'exercice de la charité. L'âme de l'héroïne, qui sera souvent évoquée tout au long du récit, lui vient donc d'outre-tombe, comme un précieux cadeau de son ami.

Très douloureuses sont les épreuves auxquelles Lise doit s'affronter. Le rôle d'opposantes correspond à deux femmes, dont l'une surtout incarne la pure méchanceté. Lydie, baronne de Rühlberg et sœur de Serge, femme veuve ruinée à la mort de son mari, habite avec ses deux fils dans la demeure de son frère, qui lui procure de forts revenus en se limitant à tolérer sa présence. Et malgré son dédain et sa jalousie envers Lise, elle sera beaucoup moins dangereuse que la véritable parente pauvre du héros : Varvara Petrowna Dougloff, cousine de Serge, dont la première apparition dans le récit, glissant silencieusement dans la salle, présente déjà les signes de l'inquiétant :

C'était une femme d'environ vingt-cinq ans, petite, maigre, légèrement contrefaite et vêtue d'une robe de soie noire toute unie. [...]

Lise lui tendit sa main, dans laquelle Varvara mit ses longs doigts aux ongles aigus, dont la vue rappela involontairement à la jeune femme les griffes d'un loup ${ }^{11}$.

\footnotetext{
${ }^{8}$ Ellen CONSTANS, « Le Premier Delly ou comment l'idéologie subvertit le récit amoureux », op. cit., p. 80.

${ }^{9}$ Ellen CONSTANS, Parlez-moi d'amour. Le roman sentimental : des romans grecs aux collections de l'an 2000, op. cit., p. 231.

${ }^{10}$ DELLY, Esclave... ou Reine?, op. cit., p. 2.

${ }^{11}$ Ibid., p. 92-93.
} 
Le premier récit de ceux qui viendront éclaircir l'intrigue de ce roman vient de la part de celle qui sera la principale adjuvante de Lise, qui se sent malheureuse et égarée. Il s'agit de la vieille Madia, ancienne nourrice de Serge, qu'elle adore toujours, et dont la connaissance du passé déchiffrera beaucoup d'énigmes. Elle racontera d'abord à Lise l'enfance du prince, élevé par son grand-père, homme cruel qui lui enseigna " la dureté de cœur, l'orgueil de sa supériorité masculine, le mépris et l'asservissement de la femme $»^{12}$. Madia est sûre que la jeune princesse est destinée à faire renaître le bon cœur du prince, et son long récit offrira d'autres informations précieuses à Lise, en la prévenant contre Varvara par des paroles prémonitoires : « Pourquoi elle vous hait? Parce qu'elle est une louve, et vous, une agnelle du bon Dieu $»^{13}$. Cette ancienne servante, presque oubliée dans un coin du château, exerce donc la fonction de certains personnages secondaires qui agissent dans les romans dellyens à la manière $\mathrm{d}^{\prime}$ ' " anges gardiens », fonction très importante dans le récit où ils apparaissent pourtant très peu :

Mais c'est justement parce qu'ils participent très sommairement à l'action qu'ils sont en mesure d'avertir le danger menaçant leurs « disciples » et de voir ce que les autres ne voient pas. Ils deviennent par là des auxiliaires du narrateur qui partagent avec lui le but de guider le lecteur dans le déchiffrement de l'intrigue ${ }^{14}$.

La condition de parente pauvre de Varvara fait d'elle un personnage monstrueux. Connaissant que la priorité de Lise est la charité, comme elle l'a démontré plusieurs fois au cours du récit, elle lui tend un piège en lui demandant de l'accompagner secourir « une pauvre famille misérable ${ }^{15}$ afin de l'enfermer dans une cabane où elle fera le terrible récit de son passé :

Il y a treize ans, une fillette arrivait avec sa mère à Kultow, et était présentée au prince Ormanoff, un tout jeune homme alors, mais aussi orgueilleux, impénétrable et dédaigneux qu'aujourd'hui. Un regard empreint de la plus indifférente froideur tomba sur l'enfant... Et pourtant, $[\ldots]$ ces yeux fascinants par leur froideur même enchaînèrent à jamais Varvara Dougloff.

12 Ibid., p. 163-164.

13 Ibid., p. 166.

${ }^{14}$ M. Carme FIGUEROLA, « Le roman dellyen et les structures du roman populaire : quelques réflexions », Le Rocambole (Bulletin des Amis du Roman populaire), Été-Automne 2011 : L'œuvre de Delly, Novelle série, nº 55-56, (pp. 91-112), p. 106.

${ }^{15}$ DELLY, Esclave... ou Reine ?, op. cit., p. 238. 
$\mathrm{Au}$ fond de son cœur, elle dressa un autel à celui qui ne daigna jamais s'apercevoir de ce culte silencieux ${ }^{16}$.

Si les métaphores animales sont fréquentes dans l'œuvre de Delly, Varvara ne s'inscrit même pas pleinement dans la ligne " des femmesanimaux livrées à des instincts mauvais ${ }^{17}$ qui, semblables au serpent du paradis, se montrent surtout comme des séductrices. Varvara, qui avoue avoir aidé l'accident qui coûta la vie à Olga et à son enfant, est, à notre avis, tout simplement sinistre. Les fréquentes comparaisons de cette femme avec une louve préfigurent cette vengeance contre Lise, dont elle ne peut même pas être la rivale. Et les loups viendront aider cette femme-louve, qui enfonce ses ongles aigus dans les bras de Lise en la poussant au dehors de la cabane en proie aux bêtes affamées. Elle sera sauvée par les gardes du prince, mais aussi, et peut-être surtout, par son premier ange gardien, Gabriel, qui a toujours veillé sur elle : «Lise comprit qu'elle était perdue, si un miracle ne la sauvait. À la pensée de la mort atroce qui se préparait, elle se sentit défaillir d'horreur, et du fond de son cœur, un appel éperdu jaillit vers le ciel... $»^{18}$.

Les récits s'enchaînent jusqu'à la fin du roman, et Catherine mourante dévoilera à sa belle-fille, en obtenant son pardon, un autre secret : sa participation dans la mort de sa mère, dont le remords l'a toujours poursuivie. La fin d'Esclave ...ou reine ? fait donc retour au secret initial, et aussi au cadre initial : le cimetière où Serge et Lise, devenus mari et femme, visitent la tombe de Gabriel. Serge est tombé amoureux de Lise le jour où elle a refusé nettement de renoncer à sa religion catholique. Et progressivement, il a cédé aux volontés de cette jeune femme qui a osé lui tenir tête dans le seul but de la rendre heureuse, de sorte que la prédiction de Madia s'est finalement accomplie : « «Croyez en la vieille Madia, Altesse : quand vous aurez quelque chose à lui demander, faites-le hardiment, et vous obtiendrez tout $»^{19}$.

\footnotetext{
${ }^{16}$ Ibid., p. 242-243.

${ }^{17}$ Ellen CONSTANS, « Cri du cœur, cri du corps, dans les romans de Delly », in Le Roman sentimental, « Trames », Limoges, 1990, (pp. 263-275), p. 273.

${ }^{18}$ DELLY, Esclave... ou Reine ?, op. cit., p. 246.

${ }^{19}$ Ibid., p. 166.
} 


\section{La centralité de l'héroïne : La fin d'une Walkyrie}

Le titre de ce roman (1916), où l'intrigue se complique au fur et à mesure que les intérêts économiques et politiques des divers personnages se succèdent, est significatif de l'anti-germanisme de Delly, qui apparaît souvent le long du récit. Brunhilde de Halweg est une femme perverse, dépourvue de tout sens moral, qui s'inscrit pleinement dans la typologie des séductrices dangereuses. Au contraire du personnage de Varvara, Brunhilde possède une grande beauté physique dont la description ne manque pas pourtant de susciter une certaine sensation d'inquiétude : "C'était une créature singulière : froide, en apparence, certainement orgueilleuse et dure, mais cependant douée d'une séduction altière, telle qu' on la peut imaginer chez les Walkyries farouches qui, dans le Walhalla, servent l'hydromel aux guerriers germains $»^{20}$. Cette walkyrie sera la rivale de l'héroïne, la naïve Aniouta Verenof, petite comtesse de 16 ans. Nous retrouvons le motif de l'orpheline qui, malgré son origine noble, se trouvant ici dépourvue de sa fortune après la mort subite de son grandpère, sera accueillie par son cousin éloigné le comte Boris Vlavesky dans sa maison seigneuriale. Le terme de parente pauvre appliqué à Aniouta revient à plusieurs reprises tout au long du texte. Et cela surtout à cause de la mère du héros, la comtesse Sophie qui, malgré la diminution de sa fortune après la mort de son mari, a éduqué son fils Boris dans le luxe, tenant surtout à conserver l'orgueil de sa caste, et n'ayant d'autre ambition que de lui faire épouser une femme riche :

Sa mère, nature froide et vaniteuse, s'était peu souciée de son éducation morale. Seuls lui importaient l'intelligence, les dons physiques très brillants, les succès mondains de ce fils unique, héritier de la vieille race. [...] Maintenant, elle n'avait plus qu'un désir, celui d'un opulent mariage qui redonnerait à Boris la situation d'autrefois ${ }^{21}$.

Cette nature de mère-marâtre se révélera nettement dans son refus d'accueillir chez elle Aniouta, cette adolescente à la santé fragile, élevée en plein air et en liberté. Néanmoins, son insistance pour la mettre en pension se

\footnotetext{
${ }^{20}$ DELLY, La fin d'une Walkyrie, Librairie Plon, Paris, 1916, p. 18-19.

${ }^{21}$ Ibid., p. 8. La mère obsédée par l'orgueil de la noblesse, dont le seul objectif dans la vie semble être la prolongation de sa lignée, constitue un type récurrent dans les romans dellyens, qui apparaîtra aussi, comme nous le verrons plus tard, dans La Maison des rossignols. Un autre roman de l'auteure que nous n'analyserons pas, Une misère dorée (1929), est aussi spécialement significatif à cet égard.
} 
heurtera dès le début à la ferme opposition de son fils, qui deviendra le tuteur de cette petite restée seule au monde. Un sentiment de sympathie réciproque s'établit dès le début entre Boris, homme expérimenté, militaire très apprécié par ses chefs, et cette jeune fille innocente qui ne connaît ni le monde ni la société. Le scepticisme du héros par rapport aux femmes commence à disparaître dès qu'il connaît Aniouta, très différente des adolescentes qu'il a rencontrées jusqu'alors : "C'était l'enfant, dans toute sa fraîcheur, dans toute son innocence. Elle était visiblement aussi à l'aise près de ce jeune et beau cousin que s'il eût été son frère $»^{22}$. Exerçant toujours le rôle de protecteur, Boris confie le soin de sa petite cousine pendant ses absences à sa chère Liouba, la vieille femme de charge de la maison depuis cinquante ans, «l'unique, la perle des perles, comme l'appelait son jeune maître $»^{23}$. Une fois de plus, nous retrouvons le rôle de la servante âgée, qui exerce ici la fonction non seulement d'adjuvante de l'héroïne mais aussi de confidente du héros, qu'elle a jadis « bercé dans ses bras plus souvent que sa propre mère ${ }^{24}$. C'est justement à cette femme que Boris dévoile ce qu'il croit être son véritable sentiment envers sa pupille : «- Il me semble que je l'ai toujours connue, cette enfant ! Je l'aime comme si elle était vraiment ma sœur $»^{25}$.

La phase de la rencontre en tant que moteur de la narration est spécialement significative, puisqu'elle présente ici « le motif de la prédestination $»^{26}$. Boris et Aniouta se reconnaissent comme frère et sœur, et ce sentiment durera longtemps. Il faudra donc, pour que le vrai sentiment de l'amour se révèle, la phase de la disjonction, des conflits, dont « la nécessité est d'autant plus grande que, souvent, les deux protagonistes ou l'un d'eux ignorent la nature de leur propre sentiment et celle de leur vis-à-vis ou se méprennent sur ce qu'ils éprouvent $»^{27}$. Plusieurs phases conflictuelles ont lieu dans ce roman, pendant lesquelles Liouba est presque le seul appui de la petite face aux deux opposantes du récit : Sophia, dont le mépris envers cette « pupille élevée par charité ${ }^{28}$ ne cessera jamais, et bien sûr Brunhilde, qui voit son orgueil blessé et ses ambitions déçues à cause de l'indifférence

\footnotetext{
${ }^{22}$ Ibid., p. 37.

${ }^{23}$ Ibid., p. 48.

${ }^{24}$ Ibid., p. 49.

${ }^{25}$ Ibid., p. 58.

${ }^{26}$ Ellen CONSTANS, «Roman sentimental, roman d'amour: Amour...toujours... », in Le Roman Sentimental, tome 2, PULIM, Limoges, 1991. (pp. 21-33), p. 30.

${ }^{27}$ Ibidem.

${ }^{28}$ DELLY, La fin d'une Walkyrie, op. cit., p. 56.
} 
manifestée par Boris à son égard. Sa malveillance s'exerce auprès de la mère du héros dont elle cherchera la complicité Et, se voyant finalement vaincue par une adolescente, elle prépare « la vengeance, plaisir des dieux- et des guerrières du Walhalla ${ }^{29}$. Vengeance inutile, du moins en ce qui concerne les deux protagonistes du roman. La seule victime de cette femme sera le cousin de Boris, Cyrille, qui, fasciné par son charme physique, l'épousera malgré les préventions du comte. Brunhilde incarne parfaitement le type des femmes qui ne sont qu'un corps :

Plus âgées que les héroïnes, ces femmes ont « vécu »; elles ont l'expérience des hommes et des choses de l'amour, expérience rarement précisée dans le texte, mais toujours connotée négativement. Elles ont donc dans la conquête de l'homme des armes ignorées des héroïnes jeunes et pures.

En vain cherche-t-on dans les romans une allusion à leur « âme »; elles n'ont qu'un corps ${ }^{30}$.

Et c'est justement parce que Brunhilde est une femme sans âme, qu'elle est, dès le début, une rivale trop faible vis-à vis de l'héroïne. Dès sa rencontre avec Boris, elle ne constitue pour lui qu'un objet de curiosité et d'observation. Par contre, Aniouta, cette jeune fille en apparence fragile, cette parente pauvre sera capable de s'attirer les sympathies non seulement de tous les domestiques - Liouba, le jardinier Yégor - mais de certains membres de la noblesse. Les sentiments charitables de l'héroïne sont cités plusieurs fois le long du récit: «On la connaissait partout, et déjà on l'aimait, la jolie petite barina, comme on l'appelait, si gracieuse pour tous, et qui, deux fois, avait su obtenir du maître des mesures d'indulgence pour de pauvres gens que l'intendant voulait expulser du domaine $»^{31}$. Aniouta saura, en effet, mener à bout la métamorphose du héros au fur et à mesure que le récit avance. C'est seulement l'héroïne qui se rend compte de la fatigue et de la santé délicate de Liouba qui, malgré son âge, ne se plaignait jamais, et elle fera voir à Boris la nécessité d'alléger son travail. Elle devient de plus en plus la « conscience vivante ${ }^{32}$ du héros jusqu'à la phase de la révélation de l'amour, qui se produira à travers la musique. Dans la deuxième partie du roman, c'est le chant d'Aniouta qui ouvre les yeux à Boris : «Aniouta !... Il l'aimait ! Depuis quand ? Peut-être depuis qu'il la

\footnotetext{
${ }^{29}$ Ibid., p. 122.

${ }^{30}$ Ellen CONSTANS: « Cri du cœur, cri du corps, dans les romans de Delly », op. cit., p. 272-273.

${ }^{31}$ DELLY, La fin d'une Walkyrie, op. cit., p. 131.

${ }^{32}$ Ibid., p. 139.
} 
connaissait $»^{33}$. Révélation de l'amour qui sera l'élément déclencheur de la métamorphose de cet homme, qui était déjà honnête et courageux : «Dieu a mis sur ma route Aniouta, et dès ce moment ma vie s'est orientée vers une voie nouvelle. Le salut m'est venu par cette enfant $»^{34}$. Le mariage aura lieu, et dans la troisième partie du roman, où le lecteur assiste à des intrigues trop compliquées, provoquées par la perversité de Brunhilde ${ }^{35}$, un personnage féminin, bien que moins important que Liouba, joue un rôle essentiel d'adjuvante : il s'agit de Rosa, une jeune fille légèrement contrefaite, femme de chambre ou plus exactement la servante des autres domestiques, comme le dit Cyrille, emprisonné par sa femme Brunhile dans un domaine secret. Rosa a toujours été la victime des mauvais traitements de tous et en particulier de Brunhilde « qui la considère comme au-dessous du plus infime animal $»^{36}$. Mais cette fille apparemment dépourvue d'intelligence, que Brunhilde destine comme servante a son mari, possède «le flair des créatures demeurées un peu primitives $»^{37}$ et, dévouée à son maître et connaissant tous les coins de ce domaine, elle guide en pleine nuit la fuite de Boris et Cyrille, manifestant, après la mort de ce dernier, tout son appui à Boris.

La mort de la Walkyrie, tuée par une balle de Boris, et son visage défiguré et affreux sont présentés comme le signe d'un châtiment mérité, où le manichéisme du roman populaire qui veut la mort des méchants rejoint l'anti-germanisme de Delly. Et cette mort, annoncée dans le titre, fait de La fin d'une Walkyrie l'un des romans dellyens où le héros « doit obtenir sa propre victoire contre le mal $»^{38}$, puisque l'on pourrait dire que Brunhilde représente une force maléfique, une instance narrative, l'incarnation du Mal qu'il faut vaincre. Et sa fonction en tant que personnage est très faible dans ce roman où l'intrigue tourne autour d'Aniouta, de sa transformation de petite fille en femme, épouse et mère. Cette héroïne, cette parente pauvre dont la seule pureté d'âme réussit à triompher de tous les obstacles, s'inscrit donc dans le type des femmes qui «ne sont nullement créés par l'Amour de l'homme,

\footnotetext{
${ }^{33}$ Ibid., p. 169.

${ }^{34}$ Ibid., p. 196.

${ }^{35}$ Daniel FROMONT signale l'inutilité dans le roman de cette dernière partie, de pure circonstance. Nous adhérons à son opinion quand il affirme que, dans cette partie « on pourrait [...] supprimer entièrement le personnage de Brunhilde, car il est inutile à l'action ». DELLY (Marie Petitjean - de la Rosière) (Avignon 1875-Versailles 1947), octobre 2003, p. 140.
}

${ }^{36}$ DELLY, La fin d'une Walkyrie, op. cit., p. 243.

${ }^{37}$ Ibid., p. 246.

${ }^{38}$ M. Carme FIGUEROLA, art. cit., p. 101. 
elles existent avant lui ; elles sont seulement sanctionnées, confirmées dans leurs qualités, couronnées $»^{39}$.

\section{De l'espace de la pauvreté à l'espace du bonheur : La Maison des Rossignols}

Les deux parties intégrantes de La Maison des rossignols (1945) sont de longueur très inégale. La première partie (ne comprenant que six chapitres), présente le caractère de l'héroïne, la ravissante petite fillette de 12 ans Lilian de Sourzy, pleine de qualités dont la plus remarquable est son incroyable capacité de travail. Son principal objectif dans la vie est de soigner sa mère malade, avec laquelle elle vit dans un très pauvre appartement de Paris depuis la mort du père, qui les a plongées dans la ruine économique. Poussée par le besoin, Emmeline, la mère de Lilian, cherche le secours de la cousine de son feu mari, lady Laurence Stanville, même si le souvenir qu'elle en garde est celui d'une femme « orgueilleuse de sa fortune, de son rang, considérant de haut ces cousins qui, bien que dans l'aisance à l'époque, demeuraient néanmoins dans une situation fort inférieure à la sienne ${ }^{40}$. Lady Stanville, qui a hérité son titre de noblesse des ancêtres de son mari, propose à Emmeline et à sa fille une hospitalité tout à fait humiliante dans sa maison de Breenwich ${ }^{41}$, en Angleterre, ce qui annonce la condition de Cendrillon à laquelle Lilian se verra de plus en plus soumise au long du roman. L'héroïne et sa mère seront logées loin des maîtres de la maison, dans des pièces froides et inconfortables, et soumises non pas à un travail mais à une servitude pire que celle des domestiques de Stanville-House. Comme Sophie dans La fin d'une Walkyrie, Lady Stanville, veuve, constitue le type de la mauvaise mère, qui a gâté son fils unique, Hugh, et qui a exalté son orgueil « en lui persuadant qu'il était un être à part, fort audessus de la commune humanité, en lui assurant que la bonté, l'indulgence, la charité n'étaient que des mots dont un Stanville ne devait pas s'occuper ${ }^{42}$. Dès leur première rencontre avec lord Stanville, Lilian et sa mère comprennent que ce jeune homme de vingt-trois ans, admiré avec dévotion par sa mère, « n'aurait qu'indifférence pour les parentes malheureuses auxquelles il daignait

\footnotetext{
${ }^{39}$ Daniel FROMONT, « Lire Delly, un plaisir toujours renouvelé », Le Rocambole (Bulletin des Amis du Roman populaire), Été- Automne 2011 : L'œuvre de Delly, Novelle série, nº 55-56, (pp. 83-90), p. 86.

${ }^{40}$ DELLY, La Maison des Rossignols, Gautier-Languereau, Paris, 1958, p. 12.

${ }^{41}$ Le nom de la ville est imaginaire. Serait-ce une déformation de Greenwich ? Une intentionnalité de déréalisation du cadre spatial semble se montrer dans ce roman.

${ }^{42}$ DELLY, La Maison des Rossignols, op. cit., p. 22.
} 
accorder l'abri de son toit $»^{43}$. En effet, Hugh gère l'usine héritée de son père d'une main de fer, insensible aux souffrances et à la misère de ses employés. Son despotisme envers Lilian éclate le jour où celle-ci le supplie de reprendre comme employé à l'usine le petit Billy qu'il a renvoyé parce qu'il arrivait parfois en retard à cause de sa mère malade. «-Que signifie cela ? ...Est-ce pour me raconter cette histoire que vous osez venir me déranger ? En vérité, voilà une belle effronterie ! ${ }^{44}$. Et, ne pouvant plus se retenir devant la cruauté de cet homme, elle lui dira : " -Vous êtes trop mauvais !... C'est affreux, ce que vous faites là $! »^{45}$. Lilian sera punie, enfermée dans sa chambre, mais elle refusera de demander pardon à Hugh. Ce passage, qui clôt la première partie du roman, montre un héros qui est encore loin de mériter le bonheur. L'influence pernicieuse de sa mère pèse encore trop sur lui. Ainsi, mère et fils se montrent d'accord sur le châtiment de Lilian, qui doit apprendre "l'humilité nécessaire à une fille que l'on élève, nourrit, entretient par charité ! ${ }^{46}$.

La différence des classes sociales est représentée dans le roman par deux espaces différents. Stanville House, demeure seigneuriale de la famille, est, malgré son opulence, un espace triste, au jardin mal soigné, et où règne le silence puisque lady Stanville déteste la musique. À cette demeure s'oppose La Maison des rossignols, l'espace de la gaieté affectueuse où habitent les O'Feilgen, des parents pauvres des Stanville méprisés par ceux-ci, comme Lilian et sa mère. Cette maison, ainsi appelée à Breenwich parce que la musique y règne tout le temps, fut autrefois un bel édifice style dix-huitième siècle, rempli d'œuvres d'art et soigneusement décoré. Mais la négligence de ses propriétaires, les Stanville, laisse tomber en ruine cette maison bâtie jadis par amour, par un Stanville «très amoureux de sa femme, une frêle et jolie Française qui languissait entre les murs de Stanville-House ${ }^{47}$, et qui deviendra souvent, non par hasard, le refuge de Lilian.

Les interventions de l'héroïne en faveur des travailleurs pauvres sont particulièrement importantes dans ce roman, d'autant plus qu'elles constituent le signe de la métamorphose du héros. Dans la deuxième partie du roman, Lilian qui, après la mort de sa mère, a démontré dans une institution sa grande capacité pour le travail, retourne malgré elle à Breenwich à l'âge de dix-huit ans. Et les interventions de la fillette devenue femme en faveur des infortunés

\footnotetext{
${ }^{43}$ Ibid., p. 26.

${ }^{44}$ Ibid., p. 47.

${ }^{45}$ Ibid., p. 48.

${ }^{46}$ Ibid., p. 50.

${ }^{47}$ Ibid., p. 37.
} 
seront de plus en plus écoutées et satisfaites par Hugh. Sa visite à la famille Osner, que Hugh veut mettre à la retraite avec une pension misérable, indigne « son âme charitable, généreuse, [...] devant le désespoir de ces pauvres gens victimes, eux aussi, de ce dur égoïsme qui avait autrefois jeté à la rue le petit Billy $»^{48}$. Cependant, à sa grande surprise, Hugh consentira à leur laisser comme retraite leur salaire entier. Et plus tard, il déclare qu'il se chargera de tous les besoins des Folken, la famille de Billy, ce qui fait que Lilian ne soit pas insensible à cette transformation :

Était-ce bien le même homme qui, six ans auparavant ?...

Mais non, elle ne voulait plus évoquer ce souvenir ! Elle devait rester sur l'impression de ce lord Stanville nouveau, qui se montrait bon, aimable... de plus en plus ${ }^{49}$.

Peu importantes sont les deux rivales de l'héroïne, spécialement Rosetta O'Feilgen, veuve d'un violoniste, jeune et belle, qui, malgré sa formation en musique, n'aspire qu'à épouser un homme riche. Plus significative est Caroline Birn, nièce du mari de lady Stanville, orpheline laide et fainéante mais riche, en qui la mère de Hugh voit l'épouse idéale pour son fils. L'hostilité de cette fille envers Lilian va s'accentuer au fur et à mesure qu'elle perçoit l'attraction de Hugh pour la parente pauvre, à qui elle prend le méchant plaisir d'imposer de lourdes tâches qui commencent à affaiblir sa santé. Mais la découverte par Hugh de la complicité entre lady Stanville et cette fille épouvantable, qu'il a toujours détestée, agit contre cette opposante qui, par sa cruauté et sa bêtise, contribue elle-même à son propre malheur et au bonheur du futur couple.

En réalité, la seule opposante véritable de l'héroïne est la mère du héros qui, par des préjugés sociaux, semble préférer pour son fils n'importe quelle épouse excepté Lilian. Mais son obstination se heurte à la volonté de son fils, qui épousera Lilian : «Oui, elle devait s'avouer vaincue... Et une larme de rage glissa lentement le long de sa joue, jusque sur le velours de son vêtement... ${ }^{50}$.

La Maison des Rossignols montre clairement «le parcours du héros vers une meilleure qualité morale $»^{51}$. À travers ce parcours, Hugh, qui est initialement très loin de la perfection, finira par mériter le bonheur. La méchante

\footnotetext{
${ }^{48}$ Ibid., p. 103.

${ }^{49}$ Ibid., 124.

${ }^{50}$ Ibid., p. 195.

51 Ellen CONSTANS, Parlez-moi d'amour. Le roman sentimental : des romans grecs aux collections de l'an 2000, op. cit., p. 232.
} 
Caroline Birn trouvera sa punition en faisant un mariage malheureux. Mais plus ambiguë est la position finale de lady Stanville. Seulement la naissance de son héritier, qui lui assure la continuité de sa caste, semble être la promesse d'une totale acceptation de sa belle-fille : «[...] elle s'assit près de lui et le contempla longuement, tandis qu'elle se répétait avec une orgueilleuse tendresse : - C'est le fils de Hugh, mon petit-fils $»^{52}$.

Final ouvert de La maison des rossignols, qui sera la nouvelle demeure de Hugh et Lilian, redevenant ainsi la maison de l'amour.

\section{Conclusion}

L'œuvre de Delly ne propose aucunement un renversement des rôles sociaux. L'inégalité des classes sociales est perçue dans ses romans comme naturelle: "L'égalité n'est pas un rêve, ni même une utopie, c'est une erreur, car cette pseudo-valeur n'est pas conforme à la volonté divine $»^{53}$. Avec la même naturalité est considérée, comme nous l'avons vu, la différence des rôles entre l'homme et la femme. Cependant, entre tradition et modernité, «Delly présente à ses lectrices, et lecteurs, une image positive du destin des femmes, un espoir, dans ce monde si masculin $»^{54}$. C'est par là que ses héroïnes exercent la fonction de salvatrices de l'homme. Capacité de travail et dévouement pour les infortunés, bonté et générosité provoquent une espèce de conversion du héros. La découverte de l'amour est liée à l'exercice de la charité et des vertus, et le mensonge et la trahison des femmes méchantes se retournent contre ellesmêmes. La Bonté est toujours récompensée, la Méchanceté toujours punie: manichéisme peut-être un peu naïf, mais qui nous renvoie au monde éternel des contes.

\footnotetext{
${ }^{52}$ DELLY, La Maison des Rossignols, op. cit., p. 201.

${ }^{53}$ Ellen CONSTANS, «Le Premier Delly ou comment l'idéologie subvertit le récit amoureux », op. cit., p. 59.

${ }^{54}$ Daniel FROMONT, « Lire Delly, un plaisir toujours renouvelé », op. cit., p. 87.
} 